\title{
Cytomegalovirus infection induces a stem cell phenotype in human primary glioblastoma cells: prognostic significance and biological impact
}

\author{
O Fornara ${ }^{1,2}$, J Bartek Jr ${ }^{1,2,3}$, A Rahbar ${ }^{1}$, J Odeberg ${ }^{1}$, Z Khan ${ }^{1}$, I Peredo ${ }^{1}$, P Hamerlik ${ }^{4}$, J Bartek ${ }^{4,5,6}$, G Stragliotto ${ }^{2}$, N Landázuri, ${ }^{\text {, 1,7 }}$ \\ and C Söderberg-Nauclér ${ }^{*, 1,7}$
}

Glioblastoma (GBM) is associated with poor prognosis despite aggressive surgical resection, chemotherapy, and radiation therapy. Unfortunately, this standard therapy does not target glioma cancer stem cells (GCSCs), a subpopulation of GBM cells that can give rise to recurrent tumors. GBMs express human cytomegalovirus (HCMV) proteins, and previously we found that the level of expression of HCMV immediate-early (IE) protein in GBMs is a prognostic factor for poor patient survival. In this study, we investigated the relation between HCMV infection of GBM cells and the presence of GCSCs. Primary GBMs were characterized by their expression of HCMV-IE and GCSCs marker CD133 and by patient survival. The extent to which HCMV infection of primary GBM cells induced a GCSC phenotype was evaluated in vitro. In primary GBMs, a large fraction of CD133-positive cells expressed HCMV-IE, and higher co-expression of these two proteins predicted poor patient survival. Infection of GBM cells with HCMV led to upregulation of CD133 and other GSCS markers (Notch1, Sox2, Oct4, Nestin). HCMV infection also promoted the growth of GBM cells as neurospheres, a behavior typically displayed by GCSCs, and this phenotype was prevented by either chemical inhibition of the Notch1 pathway or by treatment with the anti-viral drug ganciclovir. GBM cells that maintained expression of HCMV-IE failed to differentiate into neuronal or astrocytic phenotypes. Our findings imply that HCMV infection induces phenotypic plasticity of GBM cells to promote GCSC features and may thereby increase the aggressiveness of this tumor.

Cell Death and Differentiation (2016) 23, 261-269; doi:10.1038/cdd.2015.91; published online 3 July 2015

GBM is the most prevalent and the most aggressive primary malignancy of the central nervous system in adults. It is a highly vascularized and infiltrating tumor, rarely cured and prone to recurrence. The median duration of survival after diagnosis is less than 15 months, despite aggressive therapy consisting of surgical resection and concomitant radiotherapy and chemotherapy. ${ }^{1}$ Surgical resection of GBMs is typically incomplete, as they are located in the brain and are highly infiltrative. Postoperative radiotherapy and chemotherapy fail to eradicate all remaining GBM cells. Thus, a breakthrough in identifying a new treatment option leading to a cure of this disease is still lacking.

GBMs contain a subpopulation of highly tumorigenic cells with unlimited capacity for self-renewal that are commonly resistant to standard therapy. Phenotypically and functionally, these cells resemble neural stem cells and, when implanted in immunodeficient mice, can generate new tumors. As a result, they are referred to as glioma cancer initiating cells or glioma cancer stem cells (GCSCs) (reviewed in Lima et al. ${ }^{2}$ ).
Because of their apparent pivotal role in gliomagenesis and tumor recurrence after therapy, GCSCs are a major focus of research whose ultimate goal is to identify more effective therapies for GBM patients.

GCSCs were first identified by their surface expression of CD133, based on the findings that these cells grow as neurospheres under nonadherent conditions and that tumors form in vivo after implantation of only 100 CD133-positive GBM cells but not after implantation of $10^{5}$ CD133-negative GBM cells. ${ }^{3}$ The importance of CD133 as a marker of tumor aggressiveness was corroborated by the correlation between CD133 expression in brain tumors and a poor clinical prognosis. ${ }^{4-6}$ However, later studies revealed that CD133negative cells can give rise to CD133-positive cells ${ }^{7-9}$ and that both CD133-positive and CD133-negative GBM cells can initiate the development of highly aggressive tumors. ${ }^{10}$ Moreover, diverse GCSC types - all capable of self-renewal and tumor initiation - coexist within the same GBM. ${ }^{10}$ These cells often express markers associated with stem cells such as

\footnotetext{
${ }^{1}$ Karolinska Institute, Department of Medicine, Center for Molecular Medicine, Cell and Molecular Immunology, Microbial Pathogenesis Unit, Stockholm, Sweden; ${ }^{2}$ Department of Neurology and Neurosurgery, Karolinska University Hospital, Stockholm, Sweden; ${ }^{3}$ Department of Neurosurgery, Copenhagen University Hospital, Rigshospitalet, Copenhagen, Denmark; ${ }^{4}$ Danish Cancer Society Research Center, Copenhagen, Denmark; ${ }^{5}$ Department of Medical Biochemistry and Biophysics, Karolinska Institute, Stockholm, Sweden and ${ }^{6}$ Institute of Molecular and Translational Medicine, Faculty of Medicine and Dentistry, Palacky University, Olomouc, Czech Republic

*Corresponding author: N Landázuri or C Söderberg-Nauclér, Department of Medicine, Karolinska University Hospital, CMM L8:03 Cell and Molecular Immunology, Stockholm 17176, Sweden. Tel: +46 8517 79844; Fax: +46 8 313147; E-mail: natalia.landazuri@ki.se (NL) or Tel: +46 8 517 79896; Fax: +46 8 313147; E-mail:cecilia.naucler@ki.se (CS)

${ }^{7}$ These authors contributed equally to this work.

Abbreviations: GBM, glioblastoma; GCSC, glioma cancer stem cell; HCMV, human cytomegalovirus; IE, HCMV immediate early protein; GCV, ganciclovir; GSI, gamma secretase inhibitor; GFAP, glial fibrillary acidic protein

Received 08.4.15; revised 29.4.15; accepted 22.5.15; Edited by G Melino; published online 03.7.15
} 
Sox2, Notch, and Oct-4. ${ }^{11-13}$ This intratumoral heterogeneity and the resulting aggressiveness of GBMs are influenced by the location of the tumor within the brain and by tumor-associated microenvironmental factors (reviewed in Stopschinski et al. ${ }^{14}$ ). While the general validity of CD133 as a major GCSC marker is still debated and its exact function in gliomagenesis remains poorly understood, other GCSC markers have been identified, including Notch1, Oct4, Sox2, and Nestin. 4,14,15 The high levels of expression of these markers appear to functionally induce or maintain features that are characteristic of GCSCs.

We have focused on developing and testing novel treatments for GBM based on two observations: that $99 \%$ of GBMs contain human cytomegalovirus (HCMV) proteins ${ }^{16,17}$ and nucleotide sequences, and that the degree of HCMV protein expression in GBMs is a prognostic factor for patient survival. ${ }^{18}$

HCMV is a herpesvirus that infects $70-100 \%$ of the world's population. After an active primary infection, usually asymptomatic or subclinical in immunocompetent individuals, the virus establishes latency in the bone marrow and peripheral blood. Latent infections can be reactivated by inflammation. In immunocompromised individuals, primary HCMV infection and reactivation are significantly associated with morbidity and mortality. ${ }^{19,20}$

In a clinical trial, we found that the antiviral drug valganciclovir as an add-on to standard therapy led to high survival rates among GBM patients. In a retrospective analysis of patients continuously receiving such therapy for more than 6 months, the 2-year survival rate was $90 \%$ and median overall survival was 56.4 months, as compared with $18 \%$ and 13.5 months, respectively, in contemporary controls. ${ }^{17}$ These results suggest that HCMV has an oncogenic or an oncomodulatory role in GBMs, and highlight the possibility that valganciclovir may eliminate or modulate the behavior of GCSCs that may not be targeted with conventional therapies.
In light of these findings, we hypothesized that HCMV infection of GBM cells and the maintenance of a GCSC phenotype could be interrelated events. To test this hypothesis, here we investigated potential co-expression of a GCSC marker with HCMV immediate-early protein in a series of human clinical GBM specimens, and experimentally assessed the ability of HCMV infection to induce a GCSC phenotype in primary human GBM cells.

\section{Results}

CD133 and HCMV-IE are co-expressed in GBM cells and are both predictive factors for patient survival. We first analyzed, by flow cytometry, the expression of CD133 and HCMV immediate early protein (HCMV-IE) in GBM cells immediately after dissociation from fresh, surgically excised tumors obtained from 21 patients (Table 1). In 20 (95\%) of 21 GBMs $1-52 \%$ of cells expressed IE proteins (Figure 1a). In 19 of those 20 tumors, $1-70 \%$ of cells expressed CD133. In 16 tumors, $0.7-12 \%$ of cells co-expressed CD133 and HCMV-IE proteins at various levels (Figures $1 \mathrm{~b}$ and $\mathrm{c}$ ).

We next assessed the extent to which the percentage of IE-positive, CD133-positive, or IE/CD133 double-positive cells could be prognostic markers within this small cohort of GBM patients. We used the median values as cutoffs to split the patients into groups whose tumors had a high or low percentage of CD133 and IE-expressing cells. Kaplan-Meier plots showed that higher percentages of CD133-positive and IE-positive cells were associated with poor patient survival (Figures 1d and e, $P=0.0034$ for CD133, $P=0.0063$ for HCMV-IE).

The finding that both CD133 and IE are significant prognostic markers for GBM suggested that their expression was interdependent. To assess this possibility, we plotted the percentage of CD133-positive cells against the percentage of IE-positive cells in each tumor analyzed. Linear regression

Table 1 Patient characteristics

\begin{tabular}{|c|c|c|c|c|c|c|}
\hline Pt & Sex & $\begin{array}{c}\text { Age at } \\
\text { Diagnosis (years) }\end{array}$ & $\begin{array}{c}\text { TTP } \\
\text { (months) }\end{array}$ & $\begin{array}{l}\text { Survival } \\
\text { (months) }\end{array}$ & RT & $\begin{array}{l}\text { Treatment at } \\
\text { Diagnosis }\end{array}$ \\
\hline 1 & $\mathrm{~F}$ & 63 & 1 & 3 & No & Surgery, CCNU \\
\hline 2 & $\mathrm{~F}$ & 50 & 12 & 11 & Yes & Surgery, Temodal, CCNU, Avastin \\
\hline 3 & $\mathrm{~F}$ & 73 & 7 & 9 & No & Surgery, CCNU \\
\hline 4 & M & 64 & 8 & 14.5 & Yes & Surgery, Temodal, CCNU \\
\hline 5 & M & 66 & 1 & 9 & Yes & Surgery, Temodal \\
\hline 6 & $\mathrm{~F}$ & 34 & 5 & 8 & Yes & Surgery, Temodal, CCNU, Avastin \\
\hline 7 & M & 59 & 24 & 32 & Yes & Surgery, Temodal \\
\hline 8 & $\mathrm{~F}$ & 45 & 6 & 37 & Yes & Surgery, Temodal, CCNU \\
\hline 9 & $\mathrm{M}$ & 45 & 5 & 14 & No & Surgery, CCNU \\
\hline 10 & $\mathrm{M}$ & 62 & 9 & 15.5 & Yes & Surgery, Temodal, CCNU \\
\hline 11 & $\mathrm{~F}$ & 68 & 2 & 3.5 & Yes & Surgery, Temodal \\
\hline 12 & $\mathrm{M}$ & 66 & 6 & 9.5 & Yes & Surgery, Temodal \\
\hline 13 & M & 75 & 3 & 11 & No & Surgery, Temodal, CCNU \\
\hline 14 & $\mathrm{~F}$ & 64 & 16 & 27 & Yes & Surgery, Temodal \\
\hline 15 & $\mathrm{M}$ & 59 & 10 & 20 & Yes & Surgery, Temodal \\
\hline 16 & $\mathrm{~F}$ & 67 & 4 & 21 & Yes & Surgery, Temodal, CCNU \\
\hline 17 & $\mathrm{M}$ & 59 & 4 & 8 & No & Surgery, Temodal, CCNU \\
\hline 18 & $\mathrm{~F}$ & 73 & 7 & 13 & Yes & Surgery, Temodal \\
\hline 19 & $\mathrm{~F}$ & 58 & 0 & 2.5 & Yes & Surgery, Temodal \\
\hline 20 & M & 62 & 6 & 11 & Yes & Surgery, Temodal, CCNU \\
\hline 21 & M & 63 & 15 & 21 & Yes & Surgery, Temodal \\
\hline
\end{tabular}

Abbreviations: Pt, patient; $\mathrm{RT}$, radiation therapy 
a
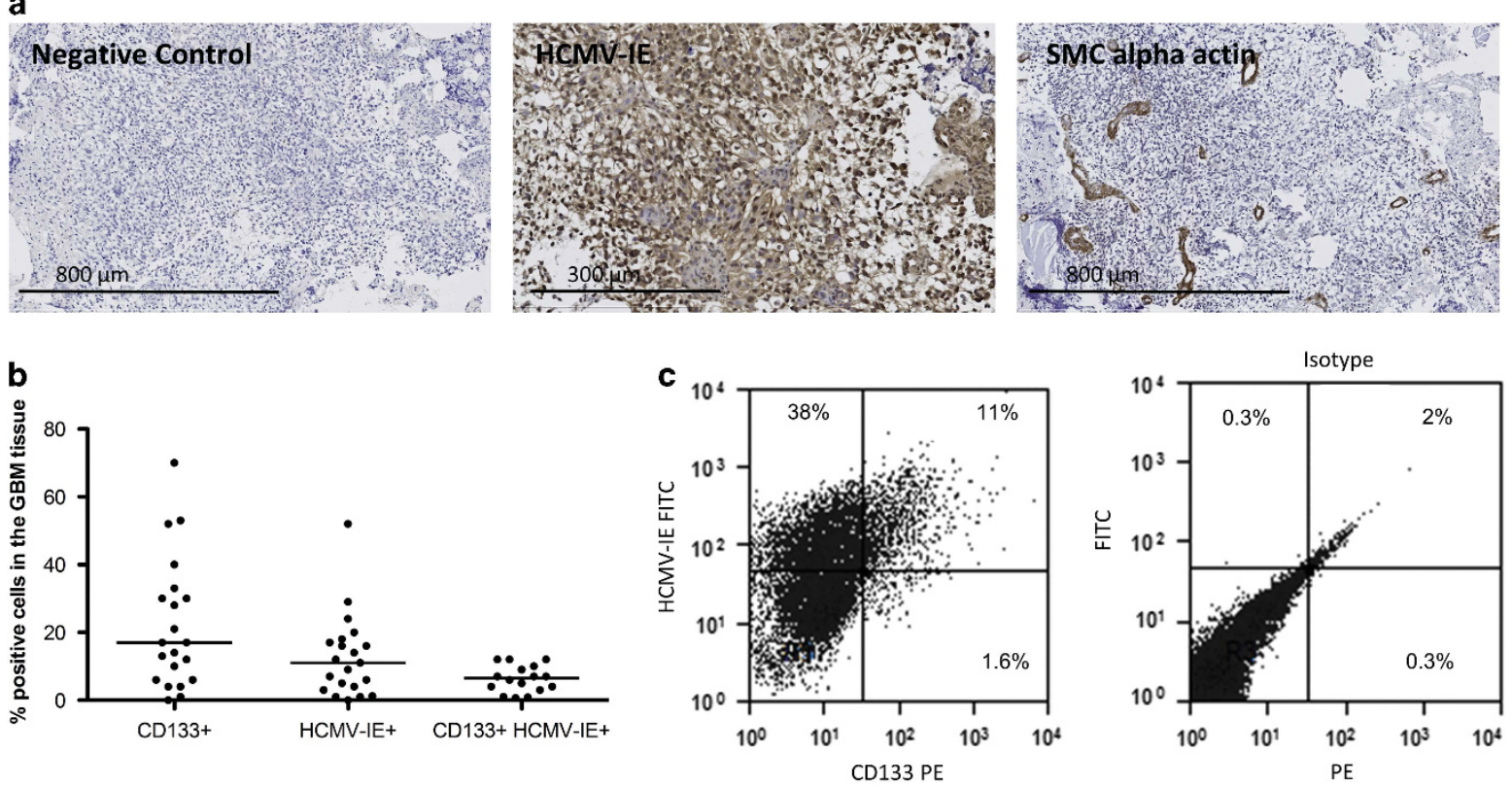

d Survival of patients with low/high amounts of CD $133+$ cells in the tumors

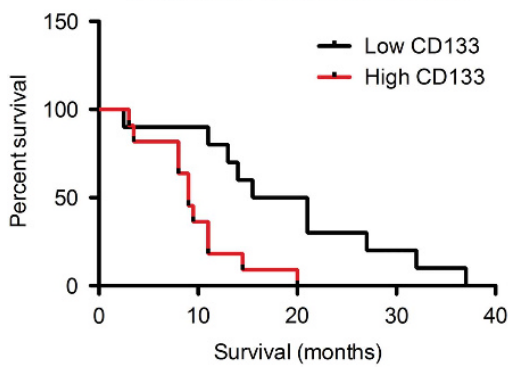

e Survival of patients with low/high amounts of HCMV-IE cells in the tumors

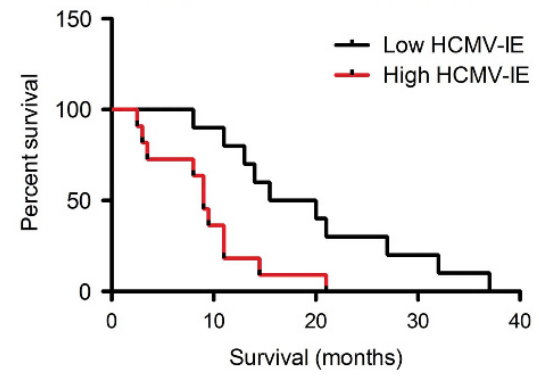

Figure 1 CD133 and HCMV-IE as prognostic markers for GBM. (a) Immunohistological staining for HCMV IE. Primary antibody was omitted from the negative control. Staining for muscle cell (SMC) alpha actin served as an isotype control. (b) Expression of CD133 and HCMV-IE in GBM tumor tissues, determined by flow cytometry (the line indicates the median percentage value). (c) Representative dot plots from flow cytometry of cells double-stained for HCMV-IE protein (FITC) and CD133 (PE). (d and e) KaplanMeier survival analysis of patients whose GBMs had high versus low expression of CD133 or HCMV-IE (The median values were used as cutoffs to split the patients into groups with high and low levels of protein expression in their tumors. $P=0.0034$ for $\mathrm{CD} 133, P=0.0063$ for HCMV-IE)

analysis showed a positive correlation $(P=0.0263$, Figure 2a). HCMV was expressed in 12-100\% of CD133-positive cells from GBM (Figure 2b). Patients with higher extent of HCMV-IE and CD133 co-expression also tended to have overall shorter survival time $(P=0.0552$, Figure $2 \mathrm{c})$.

The high degree of correlation between IE and CD133 and the high prevalence of IE expression in CD133-positive cells suggest that HCMV infection may induce or maintain a GCSC phenotype (as defined by CD133 expression) in GBM cells. Alternatively, HCMV-infected cancer cells might alter the tumor microenvironment or act in a paracrine manner to support the maintenance of CD133-positive GCSCs.

HCMV infection induces a cancer stem cell phenotype in primary GBM cells. To determine whether HCMV infection of tumor cells induces a cancer stem cell phenotype, we isolated primary GBM cells from fresh surgically excised tumor specimens and cultured them under adherent conditions. Infection of the cells with HCMV resulted in significantly increased expression of CD133 RNA and protein (Figures 3a and b). Infection also upregulated the stem cell marker Notch1 but did not affect the transcription of other stem cell markers (SOX2, Oct-4, or Nestin) (Figures $3 \mathrm{c}$ and d). The upregulation of CD133 and Notch1 indicates that HCMV infection could indeed lead to the development or maintenance of a GCSC phenotype.

Next, we assessed the ability of HCMV-infected cells to grow under nonadherent conditions and form neurospheres, a behavior typical of GCSCs. Primary GBM cells grown under adherent conditions were infected with HCMV and cultured in stem cell culture medium, which does not support adherent growth. Uninfected GBM cells served as a control. HCMV infection substantially increased the ability of GBM cells to form neurospheres (Figure 4a) and upregulated the expression of CD133, Notch 1, SOX-2, Oct-4, and Nestin (Figures $4 \mathrm{f}$ and g). However, it did not significantly affect the expression of BMI1, another GCSC maker (Figure 4g). Thus, HCMV infection of primary adherent GBM cells in vitro induced nonadherent growth of neurospheres that exhibited enhanced expression of five GCSC markers examined. Interestingly, 
a

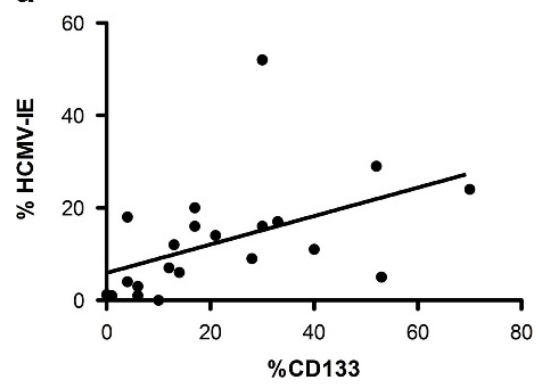

C

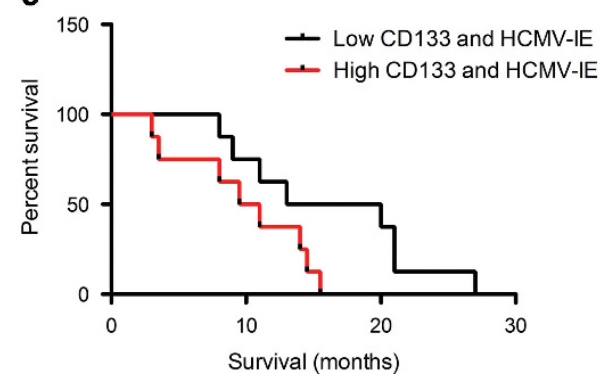

Figure 2 Correlation between CD133 and HCMV-IE expression in GBMs. (a) The percentage of CD133-expressing cells was plotted against the percentage of HCMV-IEexpressing cells in each GBM tumor analyzed. Linear regression analysis revealed a positive correlation $\left(R^{2}=0.234, P=0.0263\right)$. (b) The percentage of HCMV-IE-positive cells within CD133-positive population of GBM tumor cells, analyzed by flow cytometry (the line represents the median value). (c) Kaplan-Meier survival analysis of patients whose GBMs had high or low CD133/HCMV-IE co-expression (The median value was used as a cutoff to split the patients into two groups, $P=0.0552$ )

when working with primary cells that had been passaged eight times or more, we occasionally observed neurosphere formation without the need for previous in vitro infection with HCMV in three primary cell cultures. Few cells within these cultures displayed HCMV-IE reactivity in the cytoplasm when neurospheres were formed (Figure 4h). This staining pattern resembles that of HCMV-positive cells in GBM tissues, while cytoplasmic IE reactivity is rarely observed in in vitro infected cells where HCMV-IE is located in the nucleus.

Ganciclovir treatment is toxic to uninfected and HCMVinfected GCSCs. To confirm that neurosphere formation was due to HCMV infection, and to assess the effect of antiviral therapy on the development of this cancer stem cell phenotype, we treated the cells with ganciclovir (GCV). This drug inhibits the viral DNA polymerase and is used clinically to treat acute HCMV infection. GCV inhibited neurosphere formation and was highly toxic to both infected and uninfected GBM cells (Figures $4 b$ and c) cultured under stem cell conditions. Moreover, this cytotoxic effect preferentially affected uninfected GBM cells compared when uninfected normal MRC5 fibroblasts in culture (Figures $4 \mathrm{~d}$ and e).

A gamma secretase inhibitor blocks neurosphere formation of GCSC. The Notch pathway is important in the maintenance of GCSCs. As HCMV infection of GBM cells upregulated Notch1, we determined whether this pathway mediates the formation of neurospheres upon HCMV infection. Indeed, treatment with gamma secretase inhibitor
(GSI), which inhibits the Notch pathway, prevented neurosphere formation (Figure 4b).

HCMV infection prevents differentiation of cancer stem cells. To investigate the ability of HCMV-induced neurospheres to differentiate, we cultured them in cell culture medium that triggers differentiation of stem cells into astrocytes and neurons (Figure 5a). Remarkably, HCMVinfected GBM cells remained mostly refractory to such differentiation-inducing conditions, and the majority of infected cells did not express either neuronal or astrocytic markers (Figures $5 \mathrm{~b}$ and $\mathrm{c}$ ). These results suggested that HCMV holds GBM cells in an undifferentiated state.

\section{Discussion}

In this study, we found that the level of expression of HCMV-IE protein in freshly isolated GBM cells correlates with the level of expression of CD133, a putative marker of GCSCs. We also found that in vitro HCMV infection of primary GBM cells induces a stem cell phenotype, as determined by an increased expression of the GCSC markers CD133, Notch1, SOX2, Oct4, and Nestin, and by the acquired ability of GBM cells to grow as neurospheres under nonadherent conditions. HCMV infection also appeared to hold cells derived from neurospheres in an undifferentiated state - under culture conditions that promote their differentiation into astrocytic or neural lineages. Thus, HCMV infection of GBM cells can increase the number of GCSCs and maintain them in an undifferentiated stem-like state and thereby possibly increase the aggressiveness of GBM tumors.

Although we have consistently reported the expression of HCMV proteins and nucleic acid sequences in primary tumors, the level of endogenous expression of HCMV in primary GBM cell cultures was very low to undetectable. It is possible that the cells that are amenable to ex vivo culture under adherent conditions are less likely to harbor HCMV. Alternatively, it is possible that HCMV modulates its expression profile in a cell culture setting. In agreement with this hypothesis, we have observed fluctuating levels of HCMV-IE in cultured GBM cells. Also, after several passages (at least 8), we have occasionally observed spontaneous growth of these cells as neurospheres, without the need of in vitro infection. These cultures were then positive for HCMV-IE proteins in the cytoplasm (only in few cells). Thus, the fluctuation in the expression of HCMV-IE and the ability of cells to form neurospheres appeared to be interrelated.

GBMs are highly heterogeneous tumors featuring pronounced endogenous stress and rampant genomic instability. ${ }^{21,22}$ Four cellular phenotypes have been identified: proneural, neural, mesenchymal, and classical. ${ }^{23}$ Each phenotype has a slightly different prognosis, with the mesenchymal subtype being the most aggressive and leading to the shortest life expectancy. As shown by single-cell sequencing, all four cellular subtypes are present in each GBM, further emphasizing the complexity of this tumor type. ${ }^{24}$ Although GCSCs are present at a low frequency, they can give rise to tumors with characteristics similar to those of the primary tumor they are derived from. GCSCs have been described as tumor cells that display stem cell properties, 
a

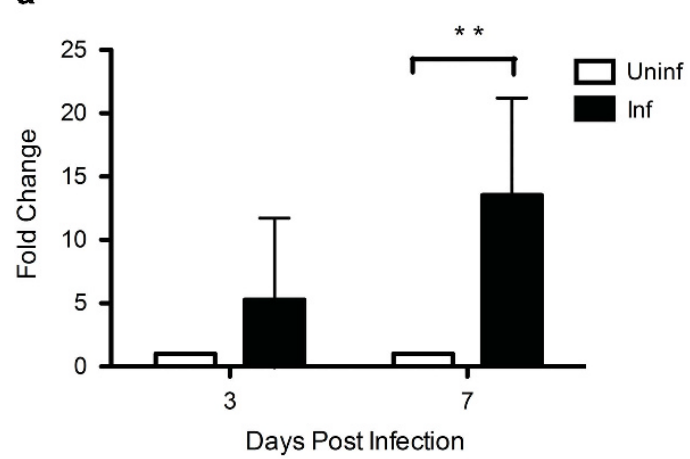

c

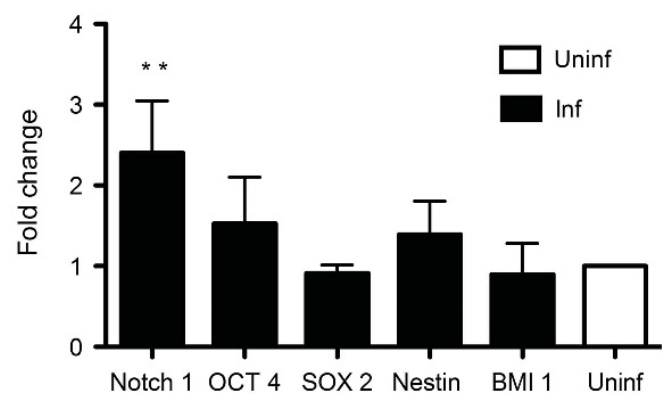

b

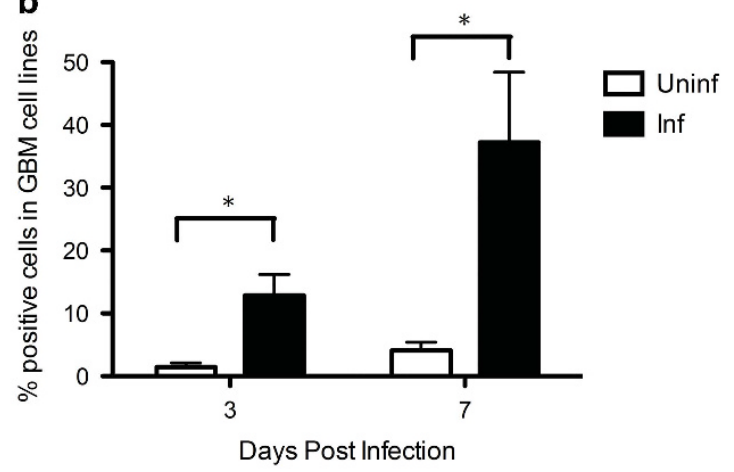

d $\quad$ Notch 1, IE, DAPI
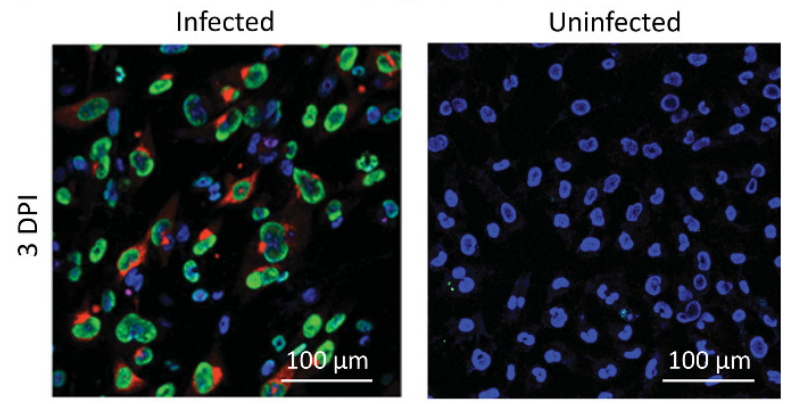

Figure 3 HCMV infection of GBM cells upregulates CD133 and Notch-1 expression. Primary GBM cells were cultured under adherent conditions and infected with HCMV in vitro. Control cells were not infected. (a) Fold change in CD133 transcription in infected cells compared with uninfected cells, assessed 3 and 7 days after infection. ${ }^{* *} P=0.0027$ versus uninfected cells. Values are mean \pm S.D. of three GBM-derived cell cultures. (b) Percentage of CD133-positive cells determined by flow cytometry 3 and 7 days after HCMV infection. ${ }^{*} P=0.028,{ }^{*} P=0.042$ versus uninfected cells. Values are mean \pm S.D. of three GBM-derived cell cultures. (c) Level of transcription of stem cell markers assessed 3 days after HCMV infection. ${ }^{*} P=0.0029$ versus uninfected cells. Values are mean \pm S.D. of three GBM-derived cell cultures. (d) Immunofluorescence staining for Notch-1 (Red), HCMV IE (green), and nucleus (DAPI, blue) 3 days after infection in GBM cells

including unlimited self-renewal, tumorigenic potential, asymmetric cell division, and multipotency - the ability to differentiate into both rapidly proliferating progenitor-like cells and more differentiated GBM cells. ${ }^{2}$ As these cells are resistant to standard therapy, they are considered a major source of GBM recurrence, which makes them a promising target for novel, more effective therapies.

The mechanisms that maintain GCSC in an undifferentiated state are highly complex and remain to be fully elucidated. Nevertheless, several pathways involving GCSC markers appear to have a major role. CD133 expression appears essential, as silencing of this protein in GBM neurospheres impairs their self-renewal and their ability to give rise to tumors in vivo. ${ }^{25}$ The Notch pathway is essential for cell-cell communication and can maintain GCSCs in an immature state by promoting their self-renewal and inhibiting their differentiation. ${ }^{12}$ Nestin overexpression increases the growth rate and migration of glioma cells and their adhesion to the extracellular matrix. ${ }^{11}$ Both SOX2 and Oct4 participate in the reprogramming of differentiated cells into induced pluripotent stem cells and are overexpressed in various types of cancer. ${ }^{13}$

HCMV infection of GBM cells upregulated all of the abovementioned markers when cells were cultured in stem cell conditions, highlighting a potential role for HCMV in the induction of GCSCs. Moreover, GBM cells grown as neurospheres and expressing HCMV-IE were unable to differentiate into astrocytic or neuronal phenotypes, highlighting a potential role for HCMV in the maintenance of GCSC state. These findings are in line with our previous results showing that HCMV infection of fetal neural progenitor inhibits their differentiation. ${ }^{26}$ Other studies have suggested that halted differentiation of neuroprogenitor cells is an early step in gliomagenesis. ${ }^{27}$ Since HCMV proteins and nucleotide sequences seem to be present in most $\mathrm{GBMs}^{16,28-30}$ and that HCMV-IE expression is a prognostic marker for poor patient survival irrespective of the GBM subtype, ${ }^{18}$ our present model of HCMV infection of primary GBM cells appears to recapitulate early events during glioma development.

Our findings suggest that HCMV has an important role in tumor progression by inducing stemness in cancer cells and maintaining a GCSC population, both of which may enhance the aggressiveness of GBMs and may confer resistance to standard therapy. Indeed, in our GBM patients, higher percentages of CD133-positive and HCMV-IE-positive cells in the tumors correlated with shorter survival. Our findings also suggest that the expression of CD133 and HCMV proteins is interdependent in GBMs.

Interestingly, we found that GCV treatment was highly toxic to both uninfected and infected GBM cells, implying that the effect of this drug is not limited to the in vitro HCMV infection of primary GBM cells. This observation may help explain why antiviral treatment of GBM patients leads to a higher survival rate than in previous studies. This drug may target GCSCs expressing HCMV proteins in vivo. Another anti-HCMV drug, cidofovir, also reduces the growth of GBMs in an animal model. The drug potentiated the effects of radiation, but the mechanism was not 
a
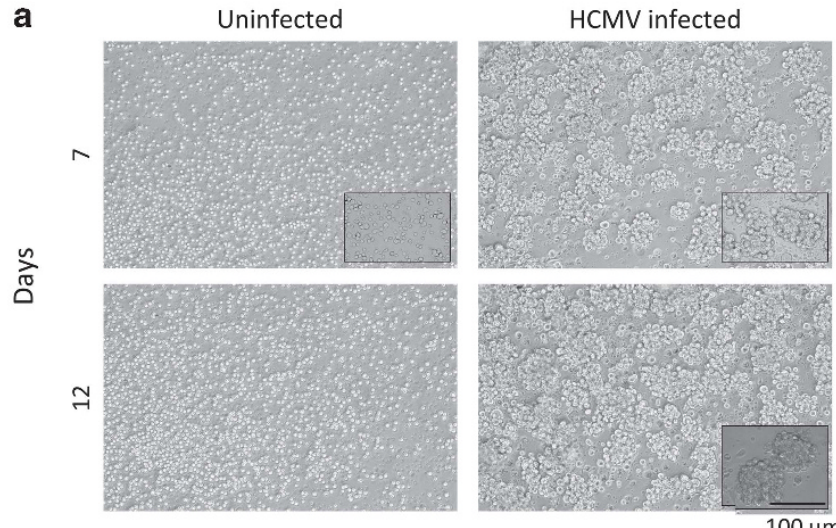

C 105
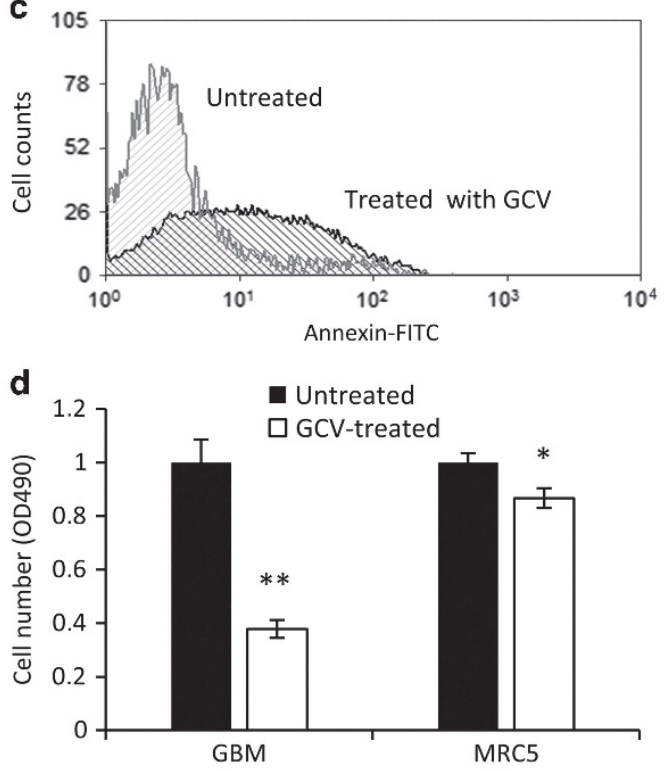

g CSC markers in GBM spheres

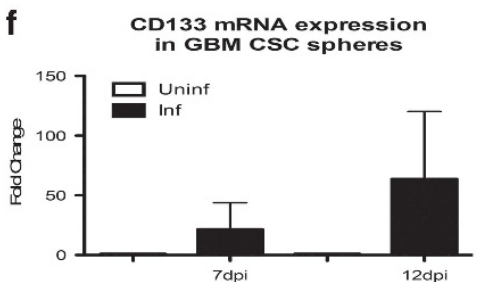

Days post CSC medium transfer

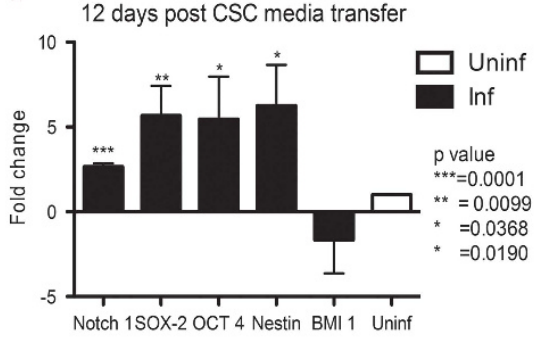

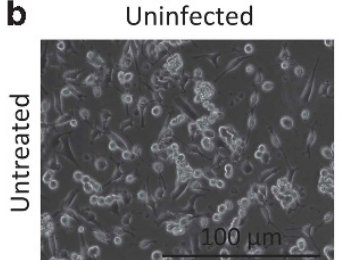
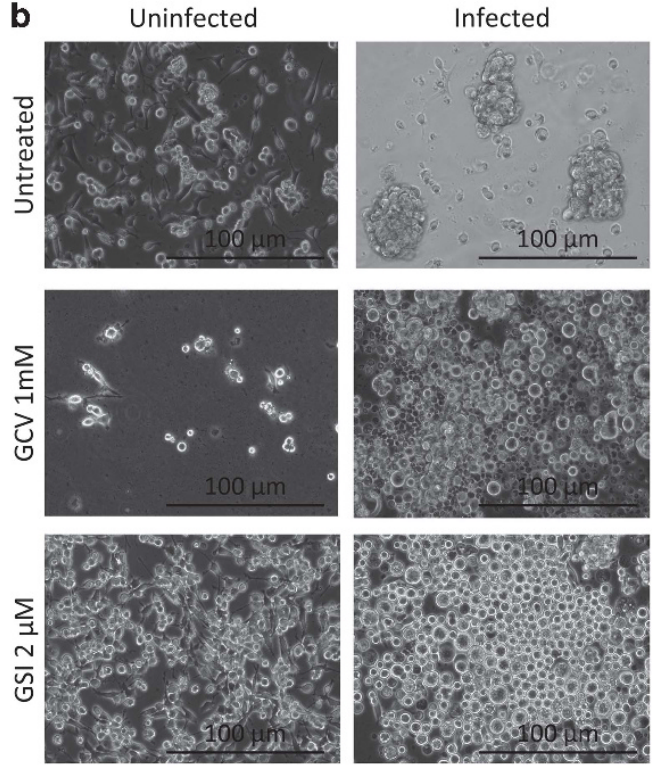

e
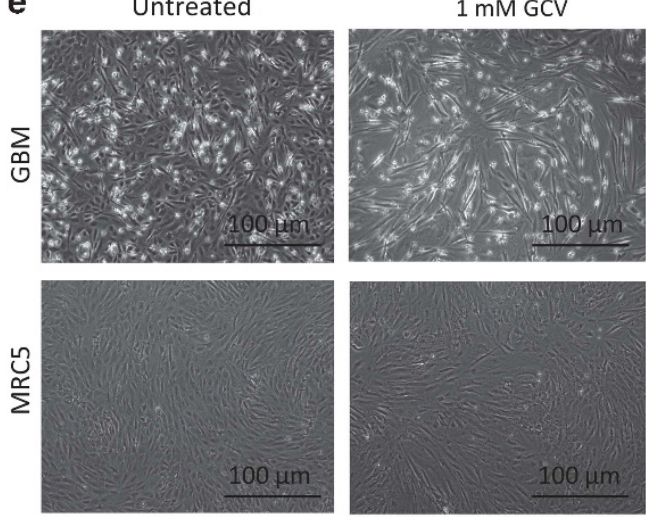

h

Isotype

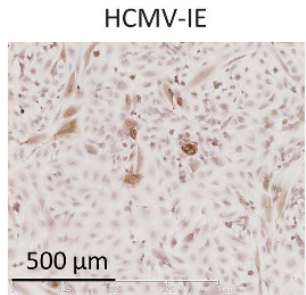

Figure $4 \mathrm{HCMV}$ infection of GBM cells induces neurosphere formation in culture. GBM cells cultured under adherent conditions were infected with HCMV and transferred to stem cell medium. (a) Light microscopic images of HCMV infected and uninfected GBM cell lines 7 and 12 days after transfer of the cells to stem cell medium. Magnification, $\times 10$. The inset shows the neurospheres at higher magnification, $\times 20$. (b) Light microscopic images of infected and uninfected cells treated with GCV or gamma secretase inhibitor (GSI) 7 days after transfer to stem cell medium. Magnification, $\times 10$. (c) Apoptosis of HCMV-infected GBM cells, untreated or treated with GCV, cultured in stem cell medium assessed by AnnexinV-FITC stain. (d) Quantification of cell viability of GBM and MRC5 cells untreated or treated with GCV for 7 days, using the MTS assay. The values are presented as mean \pm S.D. ${ }^{*} P=0.004,{ }^{\star \star} P=7.810^{-5}$. (e) Representative light microscopic images (magnification, $\times 4$ ) of GBM and MRC5 cells untreated or treated with GCV for 7 days. (f) Fold change in CD133 mRNA expression in GBM-derived neurospheres 7 and 12 days after transfer of cells to stem cell medium $(n=6)$. (g) Fold change in $\mathrm{mRNA}$ expression of the stem cell markers Notch 1, SOX2, OCT 4, Nestin, and BMl1 in neurospheres 12 days after transfer of cells to stem cell medium $(n=3){ }^{\star * \star} P=0.0001$, ${ }^{*} P=0.0099,{ }^{*} P=0.0368,{ }^{*} P=0.019$ versus uninfected cells The results are presented as mean \pm S.D. with respect to uninfected cells. (h) Representative image of cultured GBM cells at passage 8 , not infected in vitro, immunostained for HCMV-IE (brown stain) and stained with DAPI (blue nuclei) 
a

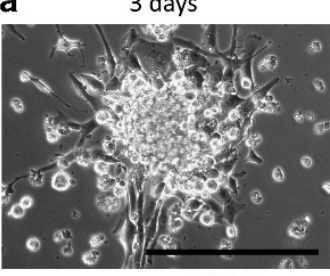

$100 \mu \mathrm{m}$

b

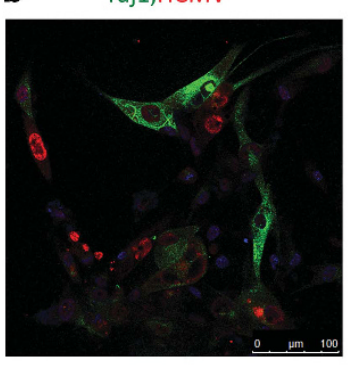

GFAP,HCMV

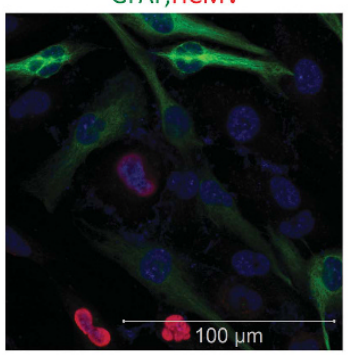

7 days

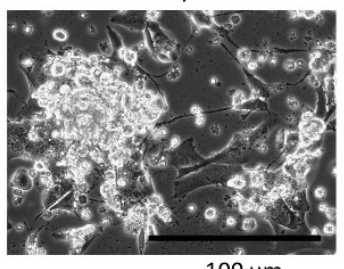

$100 \mu \mathrm{m}$

Isotype control

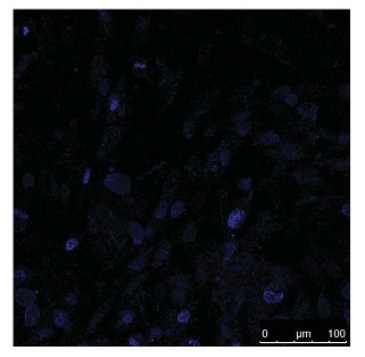

Isotype control

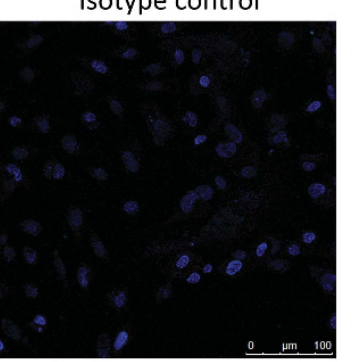

c
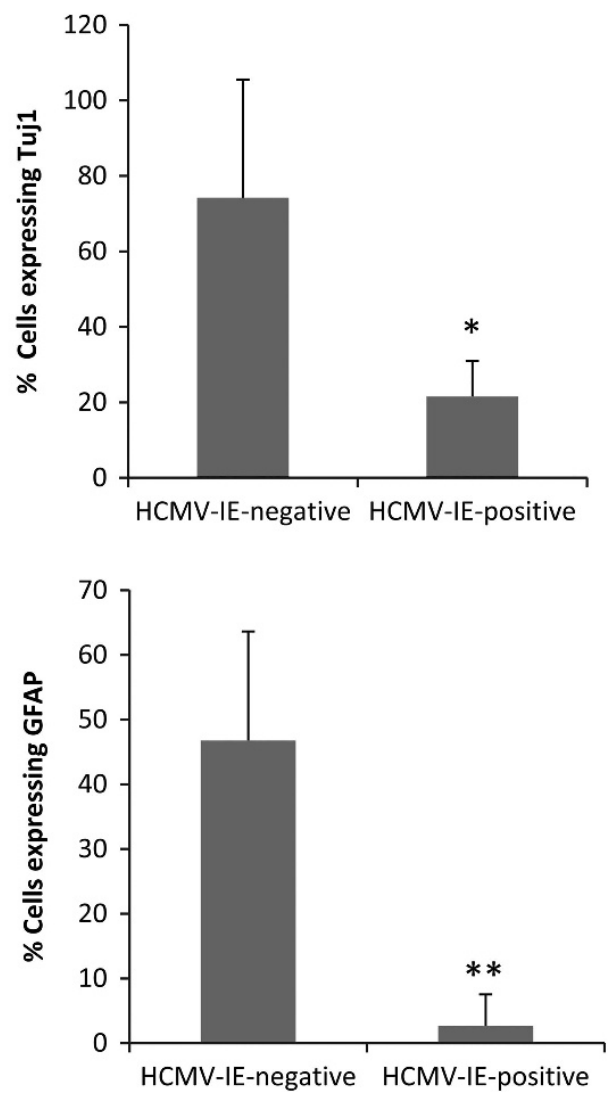

Figure 5 HCMV infection blocks GBM stem cell differentiation. Cells from neurospheres were cultured under conditions that drive differentiation into neurons and astrocytes. (a) Light microscopic images of GCSC spheres cultured under differentiation conditions at 3 and 7 days $(n=2)$ (magnification, $\times 10$ ). (b) Immunofluorescence staining of the differentiated spheres for the neuronal marker Tuj1 (green), the astrocytic marker GFAP (green), HCMV-IE (red), and nucleus (DAPI, blue). (c) Percentage of cells expressing Tuj1 or GFAP within the subpopulations of cells expressing or not expressing HCMV-IE. The values are presented as mean + S.D. ${ }^{*} P=0.0005,{ }^{\star \star} P=2.510^{-6}$

dependent on HCMV. ${ }^{31}$ Thus, antiviral therapy may be a promising alternative for treating patients with GBMs and may provide both anti-viral and off-target effects.

It is unclear how HCMV induces stemness. Recent data suggest that HCMV produces over 750 unique proteins in infected cells, which in turn can affect numerous cellular functions. ${ }^{32}$ In particular, the HCMV-IE proteins are transcription factors that can alter the expression of several viral and cellular genes. We found that HCMV-IE expression correlated with induced Notch 1 expression in adherent cells. The Notch pathway can promote self-renewal of GCSCs and inhibit their differentiation. ${ }^{12}$ In our study, treating HCMV-infected GBM cells with GSI to inhibit Notch signaling prevented neurosphere formation. Although this drug can affect other pathways, it is possible that Notch 1 downstream signaling is a key pathway controlled by HCMV to induce and maintain stemness. In fact, inhibition of Notch signaling significantly reduces GBM growth in vitro and in vivo, ${ }^{12}$ likely by reducing the size or functionality of the cancer stem cell population. ${ }^{33}$ This and other ${ }^{17,34}$ innovative strategies indicate that translational therapies targeting GCSCs could significantly improve the prognosis of patients with GBM.

In summary, we found that HCMV induces a GCSC-like phenotype in primary GBM cell lines. Our findings raise the possibility that HCMV infection of cancer cells drives a more aggressive tumor phenotype, which may explain the observed therapeutic benefit of antiviral therapy as an add-on to standard treatment for GBM patients. ${ }^{17}$ Optimal treatment of GBM most likely requires effective targeting of multiple GCSC populations expressing a range of phenotypic markers. As many of these populations are infected by HCMV, therapies targeting HCMV-infected cells should be further evaluated.

\section{Materials and Methods}

Patient tissue samples. Tumor tissues were obtained during surgical resection of GBMs in 21 patients (10 women and 11 men) at Karolinska University Hospital (Table 1). All patients had World Health Organization grade IV gliomas and received standard postoperative treatment with radiation and chemotherapy. The study was approved by the Ethics Committee at the Karolinska Institutet, Stockholm, Sweden (no. 03-220, 2006/755-31, 2008/628-31/2).

Isolation of primary GBM cell lines from GBM tumors and separation of CD133-positive cell population. Tumor tissue samples were washed in PBS, dissected to remove blood vessels and other non-tumor tissue, cut into small pieces, treated with Accutase, and filtered through $100-\mu \mathrm{m}$ nylon filter to yield a single-cell suspension. When specified, the CD133-postive cell population was enriched by magnet-assisted cell sorting with the CD133 Micro Bead Kit (Miltenyi Biotech) according to the manufacturer's guidelines.

Primary GBM cell culture and glioma stem cell culture. For adherent cultures (referred to as adherent culture conditions in the text), single-cell suspensions of primary GBMs and of normal human diploid MRC5 fibroblasts 
(obtained from ATCC) were cultured in Dulbecco's-modified Eagle's medium/F12 medium supplemented with $10 \%$ fetal bovine serum, $100 \mathrm{U} / \mathrm{ml}$ penicillin, and $100 \mu \mathrm{g} / \mathrm{ml}$ streptomycin. To stimulate the growth of cells as neurospheres, the adherent cells were trypsinized and then cultured in stem cell medium composed of serum-free Dulbecco's-modified Eagle's medium/ $\mathrm{F} 12$ supplemented with $20 \mathrm{ng} / \mathrm{ml}$ epidermal growth factor, $20 \mathrm{ng} / \mathrm{ml}$ basic fibroblast growth factor, $1 \times B 27,50 \mathrm{U} / \mathrm{ml}$ penicillin, and $50 \mu \mathrm{g} / \mathrm{ml}$ streptomycin.

Flow cytometry. Cells were stained with mouse antibody to HCMV immediateearly (IE) antigen (Argene, Biomerieux, Marcy l'Etoile, France) followed by FITCconjugated anti-mouse antibody (Life Technologies, Carlsbad, CA, USA) and with mouse PE-conjugated anti-CD133 antibody (Miltenyi Biotec, Bergisch Gladbach, Germany). The percentage of stained cells was analyzed by fluorescence-activated cell sorting with a Cyan flow cytometer (Beckman Coulter, Fullerton, CA, USA) and Summit software (v. 4.3.02, Beckman Coulter).

Viral infection. The HCMV clinical isolate VR1418 was propagated in endothelial cells and stored at $-80^{\circ} \mathrm{C}$. Viral titers were determined by plaque assays and used at a multiplicity of infection of 5-15 (optimized separately for each cell line). Approximately $70 \%$ of the cells were infected 3 days after infection and $100 \%$ of the cells were infected 7 days after infection, as determined by positive immunostaining for IE antigen. Control cells were not exposed to virus.

Treatment with GCV and GSI. GCV (Cymevene, Roche, Basel, Switzerland, $50 \mathrm{mg} / \mathrm{ml}$ ) was dissolved in water and diluted in cell culture medium to a treatment concentration of $1 \mathrm{mM}$. Infected GBMs cells were continuously exposed to $\mathrm{GCV}$, starting $2 \mathrm{~h}$ after infection. Uninfected MRC5 cells were continuously exposed to the drug, starting on the day after plating. Seven days later, cell number was estimated with the MTS cell proliferation assay (Promega, Madison, WI, USA). Similarly, GSI (Sigma Aldrich, St. Louis, MO, USA) was dissolved according to the manufacturer's guidelines and diluted in cell culture medium to a treatment concentration of $2 \mu \mathrm{M}$. Both HCMV-infected and uninfected GBM cells were continuously exposed to the drug after transfer to stem cell medium.

Apoptosis analysis. GBM-derived neurospheres treated or not treated with GCV were dissociated, and the percentage of apoptotic cells was assessed with the FITC Annexin V Apoptosis Kit (Life Technologies), according to the manufacturer's guidelines. The cells were then analyzed by fluorescence-activated cell sorting with the Cyan flow cytometer (Beckman Coulter) and the Summit v4.3.02 software (Beckman Coulter).

Immunohistochemistry and immunocytochemistry. Paraffin sections $(4 \mu \mathrm{m})$ were cut, dewaxed in xylene, and rehydrated in decreasing concentrations of ethanol. HCMV-IE antigen in the tumor tissues and in cultured cells was detected by immunohistochemistry as described. ${ }^{16,18}$ In negative control sections, the primary antibody was omitted. Smooth muscle cell alpha actin served as an isotype control. The following antibodies were used: anti-HCMV-IE (Chemicon, Merck Millipore, Darmstadt, Germany) and smooth muscle cell alpha actin (IgG2a, Biogenex, San Ramon, CA, USA).

Primary GBM cell lines were cultured and infected in chamber slides, fixed 3 days later, and incubated first with antibodies against Notch 1 (Abcam, Cambridge, UK) and IE antigen (Argene) and then with goat-anti-mouse IgG Alexa Fluor 488 (Invitrogen, Life Technologies) or goat-anti-rabbit IgG Alexa Fluor 633 (Invitrogen, Life Technologies). Cells cultured in differentiation medium were fixed, stained with antibodies against Tuj1 (Invitrogen, Life Technologies) and glial fibrillary acidic protein (GFAP) (Merck Millipore), and then stained with Alexa goat anti-mouse (Invitrogen, Life Technologies) or Alexa goat-anti-rabbit IgG (Invitrogen, Life Technologies). Isotypes for the primary antibodies were used as controls. Images of the stained cells were captured with a confocal microscope (TCS SP5, Leica) equipped with Leica Application Suite Advanced Fluorescence software (Leica Microsystems, Wetzlar, Germany). The number of cells expressing and co-expressing HCMV-IE and Tuj1 or HCMV-IE and GFAP was quantified by counting individual cells in 9 or 11 confocal microscopy images, respectively. The percentage of cells expressing Tuj1 or GFAP within the subpopulations of cells expressing or not expressing HCMV-IE was quantified.

qPCR. Total cellular RNA was isolated with the RNeasy Mini kit (Qiagen, Venlo, Limburg, Netherlands). cDNA was synthesized from equal amounts of RNA with the SuperScript III First-Strand Synthesis System (Invitrogen, Life Technologies) and
Oligo (dT) primers. Expression of CD133, Notch 1, Nestin, Oct 4, and SOX 2 was determined by TaqMan PCR with TaqPCR Master Mix (Qiagen) and Assay on Demand primers and probes (Applied Biosystems, Life Technologies), in the 7900 HT Real-Time PCR machine. The data were analyzed with SDS 2.2 software (Applied Biosystems, Life Technologies). The threshold cycle values for each gene were normalized to those of the housekeeping gene beta-2-microglobulin.

Sphere formation and differentiation. Adherent GBM primary cells were infected with HCMV and cultured under adherent conditions. Three days after infection, the cells were dissociated with $0.5 \%$ trypsin-EDTA and transferred to a new flask containing stem cell medium (as described above). Alternatively, GBM primary cells that were not infected in vitro were transferred to stem cell medium to assess the possibility of non-adherent growth as neurospheres. Twelve days later, spheres or cells dissociated from spheres were cultured in NeuroCult NS-A Differentiation Kit (Stemcell Technologies, Vancouver, BC, Canada) to induce differentiation of GCSCs to astrocytes or neurons.

Statistics. Patient survival was analyzed with Kaplan-Meier curves. Median values were used as cutoff values to define two groups of patients, which were compared by log-rank test. Differences in protein or transcript expression in infected and uninfected cells were analyzed by Student's $t$-test. In studies of cultured cells, the results are representative of at least three independent experiments with cells from GBMs of three different patients. The statistical analysis was conducted using the GraphPad Prism 5 software (GraphPad Software, La Jolla, CA, USA) or Microsoft Excel version 14.0.7147.5001 (Microsoft, Redmond, WA, USA).

\section{Conflict of Interest}

The authors declare no conflict of interest.

Acknowledgements. This work was supported by BILTEMA Foundation, Stichting af Jochnicks Foundation, Sten A Olssons Foundation for Research and Culture, Familjen Erling-Perssons Stiftelse, nexttobe, RATOS, Torsten and Ragnar Söderbergs Foundations, Dan och Jane Olssons Foundation, Swedish Cancer Foundation, Swedish Medical Research Council, Swedish Society for Medical Research (SLS), Goljes Memory Foundation, Magnus Bergvalls Foundation, Swedish Society for Medical Research (SSMF), Inga Britt och Arne Lundbergs Foundation, Danish Cancer Society, Novo Nordisk Foundation, Danish Council for Independent Research, Lundbeck Foundation, Swedish Research Council (K201499X-22627-01-4, K2013-57X-12615-16-5) and Tore Nilsons Foundation.

1. Wen PY, Kesari S. Malignant gliomas in adults. N Engl J Med 2008; 359: 492-507.

2. Lima FR, Kahn SA, Soletti RC, Biasoli D, Alves T, da Fonseca AC et al. Glioblastoma: therapeutic challenges, what lies ahead. Biochim Biophys Acta 2012; 1826: 338-349.

3. Singh SK, Hawkins C, Clarke ID, Squire JA, Bayani J, Hide T et al. Identification of human brain tumour initiating cells. Nature 2004; 432: 396-401.

4. Cheng JX, Liu BL, Zhang X. How powerful is CD133 as a cancer stem cell marker in brain tumors? Cancer Treat Rev 2009; 35: 403-408.

5. Ardebili SY, Zajc I, Gole B, Campos B, Herold-Mende C, Drmota S et al. CD133/prominin1 is prognostic for GBM patient's survival, but inversely correlated with cysteine cathepsins' expression in glioblastoma derived spheroids. Radiol Oncol 2011; 45: 102-115.

6. Zeppernick F, Ahmadi R, Campos B, Dictus C, Helmke BM, Becker N et al. Stem cell marker CD133 affects clinical outcome in glioma patients. Clin Cancer Res 2008; 14: 123-129.

7. Beier D, Hau P, Proescholdt M, Lohmeier A, Wischhusen J, Oefner PJ et al. CD133(+) and CD133(-) glioblastoma-derived cancer stem cells show differential growth characteristics and molecular profiles. Cancer Res 2007; 67: 4010-4015.

8. Ogden AT, Waziri AE, Lochhead RA, Fusco D, Lopez K, Ellis JA et al. Identification of A2B5+CD133- tumor-initiating cells in adult human gliomas. Neurosurgery 2008; 62 : 505-514; discussion 514-505.

9. Wang J, Sakariassen PO, Tsinkalovsky O, Immervoll H, Boe SO, Svendsen A et al. CD133 negative glioma cells form tumors in nude rats and give rise to CD133 positive cells. Int $J$ Cancer 2008: 122: 761-768.

10. Chen R, Nishimura MC, Bumbaca SM, Kharbanda S, Forrest WF, Kasman IM et al. A hierarchy of self-renewing tumor-initiating cell types in glioblastoma. Cancer Cell 2010; 17: 362-375.

11. Ishiwata T, Teduka K, Yamamoto T, Kawahara K, Matsuda Y, Naito Z. Neuroepithelial stem cell marker nestin regulates the migration, invasion and growth of human gliomas. Oncol Rep 2011; 26: 91-99.

12. Pannuti A, Foreman K, Rizzo P, Osipo C, Golde T, Osborne B et al. Targeting Notch to target cancer stem cells. Clin Cancer Res 2010; 16: 3141-3152. 
13. Takahashi K, Yamanaka S. Induction of pluripotent stem cells from mouse embryonic and adult fibroblast cultures by defined factors. Cell 2006; 126: 663-676.

14. Stopschinski BE, Beier CP, Beier D. Glioblastoma cancer stem cells-from concept to clinical application. Cancer Lett 2013; 338: 32-40.

15. Florio $T$, Barbieri $F$. The status of the art of human malignant glioma management: the promising role of targeting tumor-initiating cells. Drug Disc Today 2012; 17: 1103-1110.

16. Cobbs CS, Harkins L, Samanta M, Gillespie GY, Bharara S, King PH et al. Human cytomegalovirus infection and expression in human malignant glioma. Cancer Res 2002; 62 3347-3350.

17. Soderberg-Naucler C, Rahbar A, Stragliotto G. Survival in patients with glioblastoma receiving valganciclovir. N Engl J Med 2013; 369: 985-986.

18. Rahbar A, Orrego A, Peredo I, Dzabic M, Wolmer-Solberg N, Straat K et al. Human cytomegalovirus infection levels in glioblastoma multiforme are of prognostic value for survival. J Clin Virol 2013; 57: 36-42.

19. Sinclair J. Human cytomegalovirus: Latency and reactivation in the myeloid lineage. J Clin Virol 2008; 41: 180-185.

20. Soderberg-Naucler C, Fish KN, Nelson JA. Reactivation of latent human cytomegalovirus by allogeneic stimulation of blood cells from healthy donors. Cell 1997; 91: 119-126.

21. Perez-Garcia A, Carrion-Navarro J, Bosch-Fortea M, Lazaro-Ibanez E, Prat-Acin R, AyusoSacido A. Genomic instability of surgical sample and cancer-initiating cell lines from human glioblastoma. Front Biosci 2012; 17: 1469-1479.

22. Bartkova J, Hamerlik P, Stockhausen MT, Ehrmann J, Hlobilkova A, Laursen $\mathrm{H}$ et al. Replication stress and oxidative damage contribute to aberrant constitutive activation of DNA damage signalling in human gliomas. Oncogene 2010; 29: 5095-5102.

23. Verhaak RG, Hoadley KA, Purdom E, Wang V, Qi Y, Wilkerson MD et al. Integrated genomic analysis identifies clinically relevant subtypes of glioblastoma characterized by abnormalities in PDGFRA, IDH1, EGFR, and NF1. Cancer Cell 2010; 17: 98-110.
24. Patel AP, Tirosh I, Trombetta JJ, Shalek AK, Gillespie SM, Wakimoto H et al. Single-cell RNA-seq highlights intratumoral heterogeneity in primary glioblastoma. Science 2014; 344: 1396-1401.

25. Brescia P, Ortensi B, Fornasari L, Levi D, Broggi G, Pelicci G. CD133 is essential for glioblastoma stem cell maintenance. Stem Cell 2013; 31: 857-869.

26. Odeberg J, Wolmer N, Falci S, Westgren M, Seiger A, Soderberg-Naucler C. Human cytomegalovirus inhibits neuronal differentiation and induces apoptosis in human neural precursor cells. J Virol 2006; 80: 8929-8939.

27. Hu J, Ho AL, Yuan L, Hu B, Hua S, Hwang SS et al. From the Cover: Neutralization of terminal differentiation in gliomagenesis. Proc Natl Acad Sci USA 2013; 110: 14520-14527.

28. Ranganathan P, Clark PA, Kuo JS, Salamat MS, Kalejta RF. Significant association of multiple human cytomegalovirus genomic Loci with glioblastoma multiforme samples. $J$ Virol 2012; 86: 854-864.

29. Bhattacharjee B, Renzette N, Kowalik TF. Genetic analysis of cytomegalovirus in malignant gliomas. J Virol 2012; 86: 6815-6824.

30. Mitchell DA, Xie W, Schmittling R, Learn C, Friedman A, McLendon RE et al. Sensitive detection of human cytomegalovirus in tumors and peripheral blood of patients diagnosed with glioblastoma. Neuro-oncol 2008; 10: 10-18.

31. Hadaczek P, Ozawa T, Soroceanu L, Yoshida Y, Matlaf L, Singer E et al. Cidofovir: a novel antitumor agent for glioblastoma. Clin Cancer Res 2013; 19: 6473-6483.

32. Stern-Ginossar N, Weisburd B, Michalski A, Le VT, Hein MY, Huang SX et al. Decoding human cytomegalovirus. Science 2012; 338: 1088-1093.

33. Fan X, Khaki L, Zhu TS, Soules ME, Talsma CE, Gul N et al. NOTCH pathway blockade depletes CD133-positive glioblastoma cells and inhibits growth of tumor neurospheres and xenografts. Stem Cell 2010; 28: 5-16.

34. Venere M, Hamerlik P, Wu Q, Rasmussen RD, Song LA, Vasanji A et al. Therapeutic targeting of constitutive PARP activation compromises stem cell phenotype and survival of glioblastoma-initiating cells. Cell Death Differ 2014; 21: 258-269. 